\title{
Efficacy and Safety of Glecaprevir/Pibrentasvir in Korean Patients with Chronic Hepatitis C: A Pooled Analysis of Five Phase II/III Trials
}

\author{
Jeong Heo', Yoon Jun Kim², Jin-Woo Lee ${ }^{3}$, Ji Hoon Kim4, Young-Suk Lim5, Kwang-Hyub Han ${ }^{6}$, Sook-Hyang \\ Jeong ${ }^{7}$, Mong Cho ${ }^{8}$, Ki Tae Yoon ${ }^{8}$, Si Hyun Bae ${ }^{9}$, Eric D. Crown ${ }^{10}$, Linda M. Fredrick ${ }^{10}$, Negar Niki Alami ${ }^{10}$, Armen \\ Asatryan ${ }^{10}$, Do Hyun Kim ${ }^{11}$, Seung Woon Paik ${ }^{12}$, and Youn-Jae Lee ${ }^{13}$ \\ ${ }^{1}$ Department of Internal Medicine, Pusan National University College of Medicine and Medical Research Institute, Pusan National \\ University Hospital, Busan, ${ }^{2}$ Department of Internal Medicine and Liver Research Institute, Seoul National University College of \\ Medicine, Seoul, ${ }^{3}$ Department of Internal Medicine, Inha University School of Medicine, Incheon, ${ }^{4}$ Department of Internal Medicine, \\ Korea University College of Medicine, ${ }^{5}$ Department of Gastroenterology, Asan Medical Center, University of Ulsan College of \\ Medicine, ${ }^{6}$ Department of Internal Medicine, Yonsei University College of Medicine, Seoul, ${ }^{7}$ Department of Internal Medicine, Seoul \\ National University Bundang Hospital, Seoul National University College of Medicine, Seongnam, ${ }^{8}$ Department of Internal Medicine, \\ Pusan National University Yangsan Hospital, Pusan National University College of Medicine, Yangsan, ${ }^{9}$ Department of Internal \\ Medicine, College of Medicine, The Catholic University of Korea, Seoul, Korea, ${ }^{10}$ Abbvie Inc., North Chicago, IL, USA, ${ }^{11} \mathrm{AbbVie} \mathrm{Korea,}$ \\ Ltd., ${ }^{12}$ Department of Medicine, Samsung Medical Center, Sungkyunkwan University School of Medicine, Seoul, and ${ }^{13}$ Department of \\ Internal Medicine, Inje University Busan Paik Hospital, Inje University College of Medicine, Busan, Korea
}

\section{Article Info}

Received October 28, 2020

Revised December 24, 2020

Accepted January 4, 2021

\section{Corresponding Author}

Seung Woon Paik

ORCID https://orcid.org/0000-0002-6746-6652

E-mail swpaik@skku.edu

Youn-Jae Lee

ORCID https://orcid.org/0000-0003-3854-3388

E-mail yjyh0105@inje.ac.kr
Background/Aims: Glecaprevir/pibrentasvir $(\mathrm{G} / \mathrm{P})$ is the first pan-genotypic direct-acting antiviral combination therapy approved in Korea. An integrated analysis of five phase II and III trials was conducted to evaluate the efficacy and safety of G/P in Korean patients with chronic hepatitis $\mathrm{C}$ virus $(\mathrm{HCV})$ infection.

Methods: The study analyzed pooled data on Korean patients with HCV infection enrolled in the ENDURANCE 1 and 2, SURVEYOR II part 4 and VOYAGE I and II trials, which evaluated the efficacy and safety of 8 or 12 weeks of G/P treatment. The patients were either treatment-naïve or had received sofosbuvir or interferon-based treatment. Efficacy was evaluated by assessing the rate of sustained virologic response at 12 weeks posttreatment (SVR12). Safety was evaluated by monitoring adverse events (AEs) and laboratory assessments.

Results: The analysis included 265 patients; 179 (67.5\%) were HCV treatment-naïve, and most patients were either subgenotype $1 \mathrm{~B}(48.7 \%)$ or $2 \mathrm{~A}(44.5 \%)$. In the intention-to-treat population, 262 patients $(98.9 \%)$ achieved SVR12. Three patients did not achieve SVR12: one had virologic failure and two had non-virologic failures. Most AEs were grade 1/2; eight patients (3.0\%) experienced at least one grade $\geq 3 \mathrm{AE}$. No serious $A E s$ related to $\mathrm{G} / \mathrm{P}$ treatment were reported, and grade $\geq 3$ hepatic laboratory abnormalities were rare $(0.8 \%)$.

Conclusions: G/P therapy was highly efficacious and well tolerated in Korean patients with HCV infection, with most patients achieving SVR12. The safety profile was comparable to that observed in a pooled analysis of a global pan-genotypic population of patients with HCV infection who received G/P. (Gut Liver 2021;15:895-903)

Key Words: Glecaprevir and pibrentasvir; Pan-genotypic antivirals; Hepatitis C virus; Korea

\section{INTRODUCTION}

Hepatitis C virus (HCV) infection affects up to 71 million individuals worldwide and causes substantial morbidity and mortality. ${ }^{1,2}$ Patients who do not receive antiviral treatment are at high risk of serious long-term hepatic complications which include cirrhosis, decompensation, hepatocellular cancer and death. ${ }^{1,3-5}$ Among Korean patients with advanced liver diseases including liver cirrhosis and hepatocellular carcinoma, $11 \%$ to $17 \%$ present with 
HCV infection. ${ }^{6}$ The estimated seroprevalence of antiHCV antibodies in Korean adults is $0.8 \%$ (about 300,000 individuals), which increases steeply with age to $2.3 \%$ in individuals aged $\geq 70$ years. ${ }^{7.8}$ Among patients with chronic HCV infection in South Korea, virus genotypes (GTs) 1 and 2 collectively account for the vast majority (99\%) and the most common subtypes are GT1B (45\% to 59\%) and GT2A (26\% to $51 \%)$. ${ }^{6,9}$

With the advances in antiviral treatment, a cure to the $\mathrm{HCV}$ infection is achievable, which is defined as a sustained reduction in HCV RNA levels below the lower limit of quantification (LLOQ; $15 \mathrm{IU} / \mathrm{mL}$ ) at 12 or 24 weeks after completion of treatment (referred to as sustained virologic response [SVR]; SVR12 or SVR24, respectively). ${ }^{2}$ Patients who achieve SVR have a very low risk of late relapse, indicating long-term control of the infection. ${ }^{2,10}$ Until recently, the choice of treatment was based on previous treatment status, presence of cirrhosis and comorbidities, and genotyping and resistance profiles. ${ }^{11,12}$ However, the introduction of pan-genotypic combination direct-acting antiviral (DAA) regimens, using drugs with different modes of action, has simplified the care pathway for patients with HCV infection. ${ }^{3,11,13}$

Glecaprevir/pibrentasvir (G/P), a fixed-dose combination of two DAAs, is the first pan-genotypic, once-daily treatment regimen approved in South Korea for patients with chronic HCV infection regardless of genotypes. ${ }^{14,15}$ The international and Korean guidelines recommend the use of G/P therapy for both treatment-naïve and treatment-experienced patients. ${ }^{2,11,12}$

G/P therapy is also approved for a shortened 8-week treatment regimen. An integrated analysis of nine phase II and III global clinical trials, including treatment-naïve and treatment-experienced patients with chronic HCV GT1-6 infection without cirrhosis $(\mathrm{n}=2,041)$, demonstrated high rates of SVR with the G/P regimen regardless of 8-week $(98 \%)$ or 12 -week (99\%) therapy. ${ }^{16}$ In another study enrolling 343 treatment-naïve patients with chronic HCV GT16 infection and compensated cirrhosis, $97.7 \%$ of patients achieved SVR12 with 8-week G/P therapy. ${ }^{17}$

Several regional and country-level differences exist in the disease characteristics of patients with HCV infection. The screening practices, care pathways and treatment access also differ across countries. ${ }^{18}$ Hence, local data on clinical characteristics and treatment efficacy and tolerability is highly desirable, particularly in Asian countries that are generally under-represented in global clinical trials. A pooled analysis of data from six phase II and III clinical trials demonstrated high SVR12 rates with 8-week $\mathrm{G} / \mathrm{P}$ therapy in Japanese and international patients with non-cirrhotic chronic HCV GT1-2 infection $(\mathrm{n}=899) .{ }^{19}$
However, there are currently no data on the efficacy and safety of the G/P regimen in Korean subjects with $\mathrm{HCV}$ infection. Five phase II and III clinical trials of G/P therapy, including three global trials (ENDURANCE $1^{20}$ and $2^{21}$ and SURVEYOR II [part 4] ${ }^{21}$ ) and two Asian clinical trials (VOYAGE $\mathrm{I}^{22}$ and $\mathrm{II}^{22}$ ), enrolled patients from South Korea. Therefore, using pooled data from these five clinical trials, this analysis aimed to evaluate the efficacy and safety of G/P in Korean patients with GT1 or GT2 HCV infection with or without compensated cirrhosis.

\section{MATERIALS AND METHODS}

\section{Study design and population}

An integrated analysis was conducted using data from Korean patients with HCV GT1 or GT2 infection enrolled in five phase II and III trials evaluating the efficacy and safety of G/P therapy. In all five trials, patients received oral G/P therapy (300 mg/120 mg), administered as three $100 / 40 \mathrm{mg}$ tablets once daily with food, for either 8 or 12 weeks. The detailed study methodology and primary outcomes of the following registrational clinical trials have been published: ENDURANCE 1 (NCT02604017), ${ }^{20}$ ENDURANCE 2 (NCT02640482), ${ }^{21}$ SURVEYOR II part 4 (NCT02243293), ${ }^{21}$ VOYAGE I (NCT03222583) ${ }^{22}$ and VOYAGE II (NCT03235349)22 (Supplementary Table 1). All the trials required a written informed consent from enrolled patients and were designed and conducted in accordance with the ethical principles laid down in the Declaration of Helsinki, Good Clinical Practice guidelines, and applicable local regulations and were approved by independent ethics or institutional review committees.

A comparison of the inclusion and exclusion criteria across studies is described in Supplementary Table 2. For all the five trials, eligible patients were adults aged $\geq 18$ years with chronic HCV GT1 or GT2 infection (confirmed by HCV RNA $\geq 1,000 \mathrm{IU} / \mathrm{mL}$ at screening; chronic HCV infection was confirmed by anti-HCV seropositivity or plasma HCV RNA $\geq 6$ months prior to screening or liver biopsy). In addition, eligible patients were non-cirrhotic (except for VOYAGE II trial), treatment naïve or previously treated for $\mathrm{HCV}$ (interferon \pm ribavirin or sofosbuvir+ribav irin \pm interferon). Patients with prior treatment experience were required to have completed their previous HCV treatment at least 8 weeks prior to screening. The absence of cirrhosis was documented by screening FibroTest ${ }^{\oplus} \leq 0.48$, and aspartate aminotransferase platelet ratio index $<1$, or FibroScan ${ }^{\oplus}<12.5 \mathrm{kPa}$, or liver biopsy at screening. Patients with documented compensated cirrhosis were included only in the VOYAGE II trial. ENDURANCE 1, VOYAGE 
I and II allowed patients with HCV/human immunodeficiency virus (HIV) coinfection, defined as positive test result for HIV antibody at screening (HIV-1 RNA $<1,000$ copies/mL for treatment naïve or HIV-1 RNA $<$ LLOQ for those receiving antiretroviral treatment regimen)-however none of the patients enrolled in the VOYAGE trials had HIV coinfection. Hepatitis B coinfection was exclusionary for all clinical trials.

In the ENDURANCE- 1 trial, patients were randomized to receive either 8-week or 12-week treatment with G/P, whereas in the SURVEYOR II part 4 and VOYAGE I trials, patients received G/P treatment for 8 weeks. In the ENDURANCE-2 and VOYAGE II (which included patients with compensated cirrhosis), patients with GT1 or GT2 HCV infection received 12-week G/P therapy.

\section{Assessments}

For the assessment of baseline viral load and SVR12, plasma HCV RNA was quantified for each sample using COBAS AmpliPrep/TaqMan real-time reverse-transcriptase polymerase chain reaction assay (version 2.0; Roche Molecular Diagnostics, Rotkreuz, Switzerland), which has a LLOQ of $15 \mathrm{IU} / \mathrm{mL}$. HCV genotyping was done at screening using LiPA assay (Versant HCV Genotype 2.0 Assay; Siemens Healthcare Diagnostics, Erlangen, Germany) or a Sanger sequencing assay (if the LiPA assay failed) and was confirmed by phylogenetic analysis. For patients who experienced virologic failure, $\mathrm{HCV}$ resistance analysis to check for NS3 and NS5A polymorphisms was done at baseline and at the time of virologic failure using nextgeneration sequencing (detection limit: 2\%).

Adverse events (AEs) and abnormalities in physical examinations and laboratory test assessments were monitored in all five studies; the causal relationship with the study treatment $(\mathrm{G} / \mathrm{P})$ was assessed by the study investigator.

\section{Endpoints}

The primary outcome of this analysis was the efficacy of G/P therapy in Korean patients with HCV infection as assessed by SVR12, which is defined as achieving an HCV RNA level below the LLOQ $(15 \mathrm{IU} / \mathrm{mL}) 12$ weeks after the last dose of G/P. Safety evaluations included treatmentemergent AEs (occurring from day 1 of dosing until 30 days after the last dose of $\mathrm{G} / \mathrm{P}$ ) and laboratory abnormalities occurring during treatment. Secondary outcomes included incidence of on-treatment virologic failure and posttreatment relapse. On-treatment virologic failure was defined as a confirmed increase in HCV RNA of: $>1$ $\log _{10} \mathrm{IU} / \mathrm{mL}$ above nadir or $\geq 100 \mathrm{IU} / \mathrm{mL}$ after achieving HCV RNA level <LLOQ during treatment; or HCV RNA $\geq$ LLOQ at the end of treatment with $\geq 6$ weeks of treatment. Posttreatment relapse was assessed in patients who completed treatment as planned and achieved an HCV RNA $<$ LLOQ at the end of treatment, and was defined as a confirmed HCV RNA $\geq$ LLOQ between the last dose of G/P and 12 weeks thereafter. A summary of AEs and laboratory abnormalities from a previously reported pooled analysis of a global population of patients with non-cirrhotic pangenotypic HCV infection treated with G/P $(n=2,041)$ is also presented. ${ }^{16}$

\section{Statistical analysis methods}

Efficacy and safety outcomes were analyzed for the intention-to-treat population, defined as patients receiving $\geq 1$ dose of G/P (300/120 mg). The SVR12 rates and twosided $95 \%$ confidence intervals (CI; using Wilson score method) were calculated for the overall population and for subgroups based on genotype (GT1 or GT2), subgenotype (1A, 1B, 2, 2A, 2A/2C, or 2B), cirrhosis status (no cirrhosis or compensated cirrhosis), and treatment duration (8 weeks and 12 weeks). Secondary efficacy and safety endpoints were summarized with numbers and percentages of patients.

\section{RESULTS}

\section{Baseline patient demographics and disease characteristics}

This pooled analysis of data from five phase II or III clinical trials included 265 South Korean patients with chronic HCV infection who received 8-week or 12-week G/P treatment. Of these, 133 (50.2\%) were GT1-infected and 132 (49.8\%) were GT2-infected, and the most common subtypes were $1 \mathrm{~B}(48.7 \%, 129 / 265)$ and $2 \mathrm{~A}(44.5 \%, 118 / 265)$. The baseline patient demographics and disease characteristics are described in Table 1 . The median age was 60 years (range, 21 to 87 years) and $46.4 \%$ of the study population were male; the median HCV RNA level at baseline was $6.3 \log _{10}$ $\mathrm{IU} / \mathrm{mL}$ (range, 2.5 to $7.7 \log _{10} \mathrm{IU} / \mathrm{mL}$ ). Most patients were HCV treatment naïve $(67.5 \%, 179 / 265)$, without cirrhosis $(86.0 \%, 228 / 265)$, and not using injection drugs $(94.3 \%$, $250 / 265)$; more than half of the study population received 8-week G/P treatment $(57.7 \%, 153 / 265)$. The analysis population included 101 (38.1\%) patients with cardiovascular disease, 92 (34.7\%) patients with hypertension, 41 (15.5\%) patients with diabetes, and 35 (13.2\%) patients with HIV coinfection.

\section{Efficacy outcomes}

Overall, 98.9\% of patients achieved SVR12 (262/265; 
Table 1. Demographics and Disease Characteristics at Baseline in the Intention-to-Treat Population

\begin{tabular}{|c|c|c|c|}
\hline Characteristic & GT1 (n=133) & GT2 (n=132) & Overall ( $n=265$ ) \\
\hline Male sex & $63(47.4)$ & $60(45.5)$ & $123(46.4)$ \\
\hline Age, yr & 59 (21-85) & $62(25-87)$ & $60(21-87)$ \\
\hline $\mathrm{BMI}, \mathrm{kg} / \mathrm{m}^{2}$ & $23.8(17.9-36.7)$ & $24.3(17.9-33.9)$ & $24.2(17.9-36.7)$ \\
\hline \multicolumn{4}{|l|}{ HCV treatment experience } \\
\hline Naïve & $93(69.9)$ & 86 (65.2) & $179(67.5)$ \\
\hline IFN based & 39 (29.3) & 42 (31.8) & $81(30.6)$ \\
\hline SOF based & $1(0.8)$ & $4(3.0)$ & $5(1.9)$ \\
\hline \multicolumn{4}{|l|}{ Enrolled study } \\
\hline M13-590 (ENDURANCE 1) & $35(26.3)$ & 0 & 35 (13.2) \\
\hline M15-464 (ENDURANCE 2)* & $1(0.8)$ & $63(47.7)$ & $64(24.2)$ \\
\hline M14-868 (SURVEYOR II part 4) & 0 & $5(3.8)$ & $5(1.9)$ \\
\hline M15-592 (VOYAGE I)* & $74(55.6)$ & 50 (37.9) & $124(46.8)$ \\
\hline M15-593 (VOYAGE II) & $23(17.3)$ & $14(10.6)$ & $37(14.0)$ \\
\hline \multicolumn{4}{|l|}{ HCV subgenotype } \\
\hline $1 \mathrm{~A}$ & $4(3.0)$ & 0 & $4(1.5)$ \\
\hline $1 \mathrm{~B}$ & 129 (97.0) & 0 & $129(48.7)$ \\
\hline 2 & 0 & $3(2.3)$ & $3(1.1)$ \\
\hline $2 \mathrm{~A}$ & 0 & $118(89.4)$ & $118(44.5)$ \\
\hline $2 \mathrm{~A} / 2 \mathrm{C}$ & 0 & $3(2.3)$ & $3(1.1)$ \\
\hline $2 \mathrm{~B}$ & 0 & $8(6.1)$ & $8(3.0)$ \\
\hline \multicolumn{4}{|l|}{ Cirrhosis status } \\
\hline No cirrhosis & $110(82.7)$ & $118(89.4)$ & $228(86.0)$ \\
\hline Compensated cirrhosis & 23 (17.3) & $14(10.6)$ & $37(14.0)$ \\
\hline \multicolumn{4}{|l|}{ Baseline HCV RNA level, IU/mL } \\
\hline$<1.0 \times 10^{6}$ & 123 (92.5) & $118(89.4)$ & $241(90.9)$ \\
\hline$\geq 1.0 \times 10^{6}$ & 10 (7.5) & $14(10.6)$ & $24(9.1)$ \\
\hline Baseline HCV RNA level, $\log _{10} \mathrm{IU} / \mathrm{mL}$ & $6.4(2.5-7.7)$ & $6.2(2.5-7.3)$ & $6.3(2.5-7.7)$ \\
\hline \multicolumn{4}{|l|}{ IL28B } \\
\hline $\mathrm{CC}$ & $99(74.4)$ & 105 (79.5) & 204 (77.0) \\
\hline Non-CC & $34(25.6)$ & 27 (20.5) & $61(23.0)$ \\
\hline \multicolumn{4}{|l|}{ History of disorders } \\
\hline Diabetes & 23 (17.3) & $18(13.6)$ & $41(15.5)$ \\
\hline Depression or bipolar disorder & $6(4.5)$ & $7(5.3)$ & $13(4.9)$ \\
\hline Bleeding disorder & $1(0.8)$ & $1(0.8)$ & $2(0.8)$ \\
\hline Hypertension & $48(36.1)$ & 44 (33.3) & $92(34.7)$ \\
\hline Cardiovascular disease & 51 (38.3) & 50 (37.9) & $101(38.1)$ \\
\hline HIV coinfection & $35(26.3)$ & 0 & 35 (13.2) \\
\hline \multicolumn{4}{|l|}{$\mathrm{G} / \mathrm{P}$ treatment duration } \\
\hline 8 Weeks & 98 (73.7) & $55(41.7)$ & $153(57.7)$ \\
\hline 12 Weeks & 35 (26.3) & 77 (58.3) & $112(42.3)$ \\
\hline \multicolumn{4}{|l|}{ History of injection drug use } \\
\hline Yes & $7(5.3)$ & $8(6.1)$ & $15(5.7)$ \\
\hline No & $126(94.7)$ & 124 (93.9) & 250 (94.3) \\
\hline
\end{tabular}

Data are presented as number (\%) or median (range).

GT, genotype; BMI, body mass index; HCV, hepatitis C virus; IFN, interferon; SOF, sofosbuvir; IL28B, interleukin 28B; HIV, human immunodeficiency virus; G/P, glecaprevir/pibrentasvir.

*Including patients who received placebo during the double-blind treatment period followed by G/P during the open-label treatment period.

95\% CI, 96.7 to 99.6) (Fig. 1). The proportions of patients with GT1 and GT2 achieving SVR12 were 99.2\% (132/133; 95\% CI, 95.9 to 99.9$)$, and $98.5 \%$ (130/132; $95 \%$ CI, 94.6 to 99.6), respectively. All patients with compensated cirrhosis (included in VOYAGE II trial and received 12-week G/P therapy) achieved SVR12 (100\%, 37/37); among patients without cirrhosis, $98.7 \%$ (225/228; $95 \%$ CI, 96.2 to 99.6$)$ achieved SVR12 (Table 2). All patients with subgenotypes $1 \mathrm{~A}, 2,2 \mathrm{~A} / 2 \mathrm{C}$ and $2 \mathrm{~B}$ (Fig. 2A), and with a 12-week treatment duration achieved SVR12, whereas, 98.0\% (150/153; 95\% CI, 94.4 to 99.3 ) of subjects with 8-week treatment duration achieved SVR12 (Fig. 2B).

A total of three patients did not achieve SVR12, including one virologic failure and two non-virologic failures 


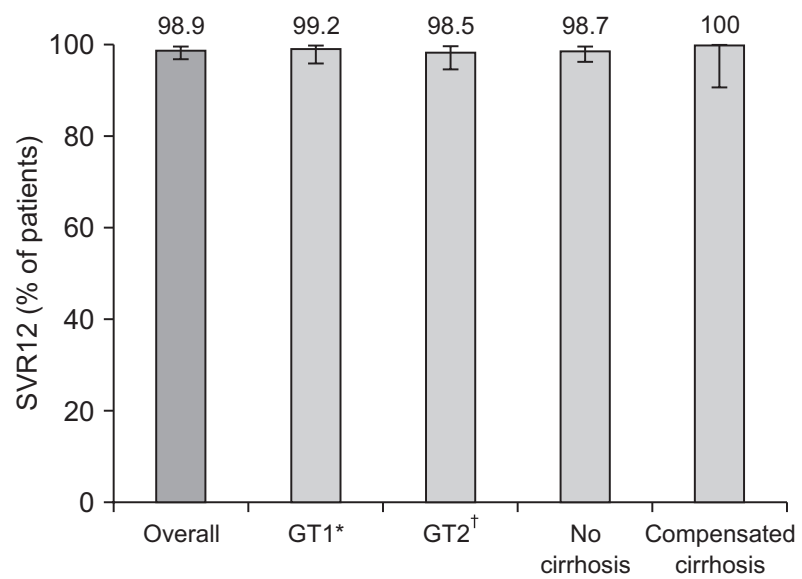

Fig. 1. Number and percentage of Korean subjects with SVR12 in the intention-to-treat population. Rates of SVR12 by genotype, cirrhosis status and treatment duration ( 8 or 12 weeks). Error bars represent $95 \%$ confidence intervals.

GT, genotype; SVR12, sustained virologic response at 12 weeks posttreatment. *4 Patients were GT1A and 129 patients were GT1B; ${ }^{\dagger} 118$ Patients were GT2A, 8 were GT2B, 3 were GT2A/2C, and 3 had unknown subtype.
(Table 3). Two patients with GT2A infection did not achieve SVR12; one patient experienced virologic failure due to posttreatment relapse at 12 weeks, and another patient was missing HCV RNA data in the SVR12 analysis window. One patient with GT1B infection experienced hyperbilirubinemia leading to premature discontinuation of $G / P$ treatment, which was assessed as not related to the treatment by the study investigators. All three patients who did not achieve SVR12 were non-cirrhotic, had no HIV coinfection, and received 8-week G/P therapy.

We also analyzed the long-term follow-up data (as of date/month/year) available from the included trials for the incidence of relapse or reinfections. None of the Korean patients included in the trials experience relapse or reinfections. Overall, there was only one case of reinfection in SURVEYOR II part 4 study in a non-Korean participant.

\section{Safety outcomes}

Overall, 119 patients (44.9\%) experienced an AE, with

Table 2. Primary (SVR12) and Secondary (on-Treatment Virologic Failure and Posttreatment Relapse) Outcomes in the Intention-to-Treat Population

\begin{tabular}{|c|c|c|c|c|c|c|c|}
\hline \multirow[b]{2}{*}{ Outcome } & \multicolumn{3}{|c|}{ No cirrhosis (n=228) } & \multicolumn{3}{|c|}{ Compensated cirrhosis (VOYAGE II) (n=37) } & \multirow{2}{*}{$\begin{array}{l}\text { Overall } \\
\text { (n=265) }\end{array}$} \\
\hline & $\begin{array}{l}\text { All patients } \\
\quad(n=228)\end{array}$ & $\begin{array}{c}\text { GT1 } \\
(n=110)\end{array}$ & $\begin{array}{c}\text { GT2 } \\
(n=118)\end{array}$ & $\begin{array}{l}\text { All patients } \\
\quad(n=37)\end{array}$ & $\begin{array}{c}\text { GT1 } \\
(n=23)\end{array}$ & $\begin{array}{c}\text { GT2 } \\
(n=14)\end{array}$ & \\
\hline SVR12 & 225 (98.7) & $109(99.1)$ & 116 (98.3) & $37(100)$ & $23(100)$ & $14(100)$ & 262 (98.9) \\
\hline \multicolumn{8}{|l|}{ Virologic failure } \\
\hline On-treatment & 0 & 0 & 0 & 0 & 0 & 0 & 0 \\
\hline Relapse* & $1(0.4)$ & 0 & $1(0.8)$ & 0 & 0 & 0 & $1(0.4)$ \\
\hline \multicolumn{8}{|l|}{ Non-virologic failure } \\
\hline Premature G/P discontinuation & $1(0.4)$ & $1(0.9)$ & 0 & 0 & 0 & 0 & $1(0.4)$ \\
\hline Missing SVR12 data & $1(0.4)$ & 0 & $1(0.8)$ & 0 & 0 & 0 & $1(0.4)$ \\
\hline
\end{tabular}

Data are presented as number (\%).

SVR12, sustained virologic response at 12 weeks posttreatment; GT, genotype; G/P, glecaprevir/pibrentasvir.

*Among patients who completed treatment as planned and achieved an hepatitis C virus RNA level less than lower limit of quantification at final treatment visit and had posttreatment data $(n=262)$.
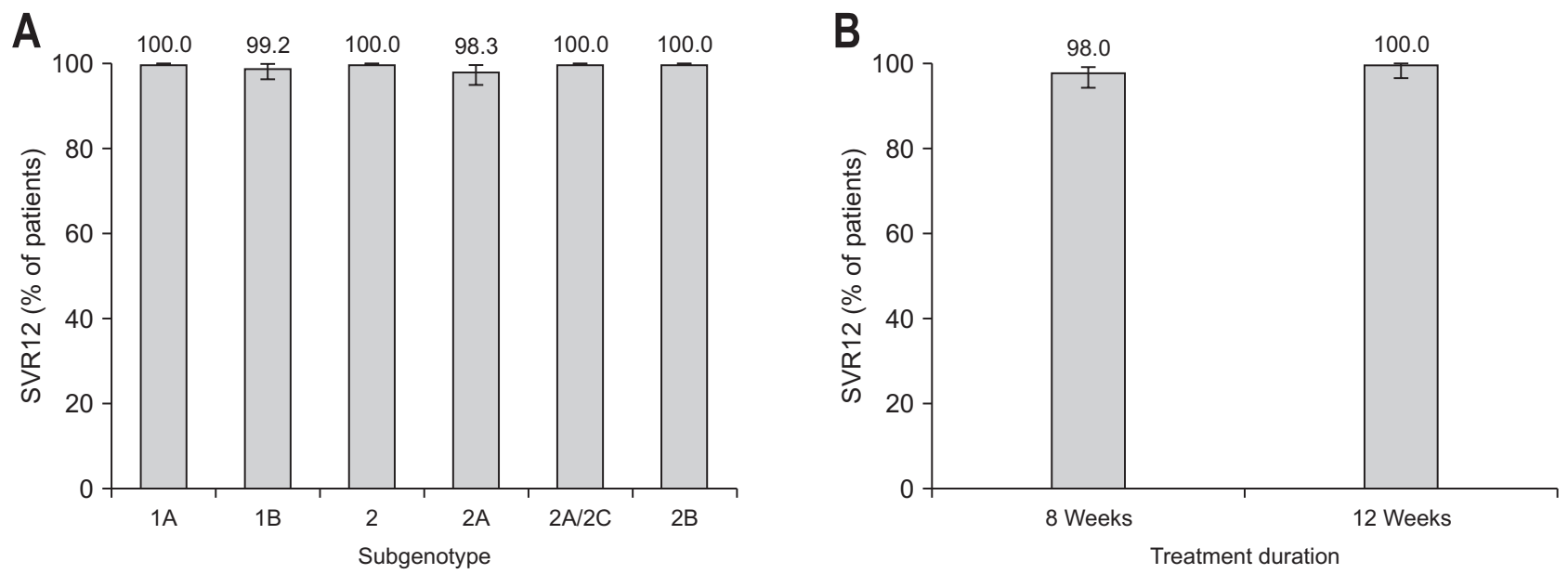

Fig. 2. Number and percentage of Korean subjects with SVR12 by subgroup in the intention-to-treat population. Rates of SVR12 by (A) subgenotype $(1 \mathrm{~A}, 1 \mathrm{~B}, 2,2 \mathrm{~A}, 2 \mathrm{~A} / 2 \mathrm{C}$, and $2 \mathrm{~B})$ and (B) G/P treatment duration (8 weeks and 12 weeks). Error bars represent $95 \%$ confidence intervals. SVR12, sustained virologic response at 12 weeks posttreatment; G/P, glecaprevir/pibrentasvir. 
Table 3. Characteristics of Patients Experiencing Virologic and Non-Virologic Failure at Baseline and the Time of Failure from the VOYAGE I Clinical Trial

\begin{tabular}{|c|c|c|c|}
\hline Characteristic & Patient 1 & Patient 2 & Patient 3 \\
\hline Reason for nonresponse & Relapse & Missing SVR12 data* & Premature G/P discontinuation \\
\hline Sex/age, yr & Male/66 & Female/74 & Female/77 \\
\hline Treatment history & SOF-experienced & Naïve & Naïve \\
\hline HCV subtype & $2 \mathrm{~A}$ & $2 \mathrm{~A}$ & $1 \mathrm{~B}$ \\
\hline Compensated cirrhosis & No & No & No \\
\hline Baseline HCV RNA level, IU/mL & $8.6 \times 10^{6}$ & $21 \times 10^{6}$ & $13.1 \times 10^{6}$ \\
\hline IL28B subtype & $\mathrm{CC}$ & $\mathrm{CC}$ & $\mathrm{CC}$ \\
\hline Treatment duration, wk & 8 & 8 & 8 \\
\hline HIV coinfection & No & No & No \\
\hline \multicolumn{4}{|l|}{ NS3 variants ${ }^{\dagger}$} \\
\hline Baseline & None & None & S122T, V170I \\
\hline At failure & None & NA & NA \\
\hline \multicolumn{4}{|l|}{ NS5A variants ${ }^{\ddagger}$} \\
\hline Baseline & L31M & T24A, L31M & Q54Y, Q62D, Y93H \\
\hline At failure & L31M, C92S & NA & NA \\
\hline
\end{tabular}

HCV, hepatitis C virus; IL28B, interleukin 28B; HIV, human immunodeficiency virus; NS, nonstructural protein; SVR12, sustained virologic response at 12 weeks posttreatment; G/P, glecaprevir/pibrentasvir; SOF, sofosbuvir; NA, not available.

*No detectable HCV RNA at last visit (posttreatment week 4, posttreatment day 27); did not return for SVR12 visit. Baseline polymorphisms and treatment-emergent substitutions at signature amino acid positions: ${ }^{\dagger} \mathrm{NS} 3(36,43,54,55,56,80,107,122,155,156,158,168,170,175)$ and ${ }^{\ddagger} \mathrm{NS} 5 \mathrm{~A}$ $(24,28,29,30,31,32,54,58,62,92,93)$ at $2 \%$ detection threshold.

Table 4. Overview of AEs and Laboratory Abnormalities in the Intention-to-Treat Population

\begin{tabular}{lcc}
\hline \multicolumn{1}{c}{ AE } & Korean population $(n=265)$ & Pooled analysis of global phase $\|\&\| I^{\ddagger}(n=2,041)$ \\
\hline Any AE & $119(44.9)$ & $1,343(65.8)$ \\
Serious AE & $7(2.6)^{*}$ & $42(2.1)$ \\
G/P-related AE & $30(11.3)$ & $N A$ \\
G/P-related serious AE & 0 & $1(<0.1)$ \\
AE leading to G/P discontinuation & $1(0.4)$ & $10(0.5)$ \\
AEs in $\geq 10 \%$ of all patients & $0^{+}$ & $344(16.9)$ \\
Headache & & $281(13.8)$ \\
Fatigue & & $1(<0.1)$ \\
Laboratory abnormalities & $1(0.4)$ & $7(0.3)$ \\
ALT, grade $\geq 3(>5 \times U L N)$ & 0 & $7(0.3)$ \\
AST, grade $\geq 3(>5 \times U L N)$ & $1(0.4)$ & \\
Total bilirubin, grade $\geq 3(>3 \times U L N)$ & &
\end{tabular}

Data are presented as number (\%). This table is presenting "data in perspective" from two separate analyses. ${ }^{16}$

$A E$, adverse event; G/P, glecaprevir/pibrentasvir; ALT, alanine aminotransferase; AST, aspartate aminotransferase; ULN, upper limit of normal; NA, not available.

*Bile duct stone ( $n=2)$, followed by urinary tract infection, radius fracture, ligament rupture, papillary thyroid cancer, and depression ( $n=1$ each); ${ }^{\dagger}$ Five most frequent AEs: upper respiratory tract infection (7.2\%), fatigue (4.2\%), diarrhea (3.0\%), headache (2.6\%), and pruritus $(2.3 \%)$; ${ }^{\ddagger}$ This is not a comparison performed in the current study of Korean patients.

most being grade 1 or 2; eight patients (3.0\%) reported at least one AE of grade 3 or higher severity (Table 4 ). AEs related to study treatment were reported in 30 patients (11.3\%); none of these AEs were of grade 3 or higher severity. Serious AEs occurred in seven patients (2.6\%) and were unlikely to be related to G/P therapy as assessed by the investigator: bile duct stone $(0.8 \%)$, urinary tract infection $(0.4 \%)$, radius fracture $(0.4 \%)$, ligament rupture $(0.4 \%)$, papillary thyroid cancer $(0.4 \%)$, and depression $(0.4 \%)$. The most commonly reported AEs were upper respiratory tract infection $(7.2 \%)$, fatigue $(4.2 \%)$, diarrhea $(3.0 \%)$ headache $(2.6 \%)$, and pruritus $(2.3 \%)$. Only one patient $(0.4 \%)$ experienced an $\mathrm{AE}$ which led to treatment discontinuation (blood bilirubin increased); this AE was considered not related to G/P. The incidence of laboratory abnormalities of grade $\geq 3$ severity was rare and none of the patients experienced grade $\geq 3$ abnormalities in aspartate aminotransferase levels. Only two patients experienced grade 3 abnormalities in bilirubin and alanine aminotransferase, respectively.

The safety profile of G/P therapy in the Korean population was comparable with that observed in the integrated analysis of nine global phase II and III clinical trials in which patients with non-cirrhotic HCV GT1-6 
infection were treated with G/P therapy (Table 4$){ }^{16}$ The incidence of AEs was slightly lower in the Korean population $(44.9 \%, 119 / 265)$ than in the global pooled analysis $(65.8 \%, 1,343 / 2,041)$. Serious AEs were reported in 2.6\% $(7 / 265)$, and $2.1 \%(42 / 2,041)$ of patients in the Korean and global pooled analyses, respectively. Serious AEs related to treatment occurred only in one patient in the global pooled analysis. AEs leading to drug discontinuation were reported in $0.4 \%(1 / 265)$, and $0.5 \%(10 / 2,041)$ of patients in the Korean and global pooled analyses, respectively. Among the Korean population, no AEs occurred in $\geq 10 \%$ of the subjects whereas in the global pooled analysis, the most common AEs were headache (16.9\%), and fatigue (13.8\%). Incidence of grade 3 or higher hepatic laboratory abnormalities was low in both Korean and global analyses (alanine aminotransferase $0.4 \%$ and $<0.1 \%$; aspartate aminotransferase $0 \%$ and $0.3 \%$; and total bilirubin $0.4 \%$ and $0.3 \%$, respectively)

\section{DISCUSSION}

The findings from this pooled analysis demonstrated high efficacy of 8-week or 12-week G/P therapy in Korean patients with HCV infection, regardless of genotype, subgenotype, presence of cirrhosis, and treatment duration. As in the global integrated analysis, G/P therapy was well tolerated in Korean patients with HCV infection, with low incidence of serious AEs or AEs leading to treatment discontinuation. Findings from this analysis of Korean patients also indicate that the study population and disease characteristics were representative of Korean patients with HCV; for instance, genotypes GT1B and GT2A were the most common subtypes, accounting for $93 \%$ of all patients. $^{9}$

This is the first analysis evaluating the efficacy and safety of G/P in Korean HCV GT1- or GT2-infected patients with or without compensated cirrhosis. HCV genotypes appear to be a key predictive factor of liver disease severity and treatment response, with a distinct geographical distribution. Among the Korean population GT1 and GT2 account for $99 \%$ of $\mathrm{HCV}$ infection. ${ }^{6}$ Previous studies investigating treatment response in Korean patients with $\mathrm{HCV}$ infection have shown high therapeutic efficacy in this population, even before the introduction of new pan-genotypic DAA agents. For example, results from a retrospective study evaluating the efficacy of pegylated interferon and ribavirin therapy in Korean patients with chronic HCV infection showed a superior therapeutic efficacy of this regimen in Koreans as compared with Caucasians, with overall SVR rates ranging between $63 \%$ and $81 \% .{ }^{23}$ Even though the evidence on the efficacy of first-generation DAA in Korean patients with HCV infection is very limited, ${ }^{11}$ data from a real-world study conducted in Korean patients demonstrated high SVR12 rates of $93 \%$ with the firstgeneration DAA treatment combination daclatasvir plus asunaprevir. ${ }^{24}$ Another study comparing the efficacy of several DAA regimens in Asian patients with HCV showed SRV12 rates of $99.2 \%$, and a significant improvement in quality of life with ledipasvir/sofosbuvir combination, the previous standard treatment for HCV GT1A infection. ${ }^{25}$ Furthermore, a retrospective study evaluating sofosbuvirbased therapy showed an SVR12 rate as high as 100\% in Korean patients with $\mathrm{HCV}$ infection. ${ }^{26}$

Results from the current study demonstrated high efficacy of an 8-week regimen of the pan-genotypic G/P therapy, which can have significant implications on the treatment burden and care pathway for patients with HCV. For example, the G/P regimen as first-line therapy would reduce the treatment duration without compromising efficacy. Shortened treatment duration also implies less ontreatment monitoring and physician visits, enabling costreductions in terms of reduced number of diagnostic procedures and clinic visits, thus improving the accessibility of treatment for a larger number of patients. In addition, as $\mathrm{G} / \mathrm{P}$ is effective across all genotypes, use of this regimen may preclude baseline assessments, such as genotyping. The promotion of a simplified pretreatment evaluation in patients with HCV GT1 or GT2 infection receiving the $\mathrm{G} / \mathrm{P}$ pan-genotypic regimen would help prevent liver disease complications and further HCV transmission; thereby contributing towards $\mathrm{HCV}$ elimination. ${ }^{27}$ However, genotyping is still considered as an essential component of HCV diagnosis in South Korea. ${ }^{9}$

The incidence of AEs was slightly lower in the Korean population than in the global integrated analysis, $44.9 \%$ $(119 / 265)$ versus $65.8 \%(1,343 / 2,041)$; however, statistical analysis of this difference was not performed. ${ }^{16}$

Limitations of this pooled analysis include small sample size $(\mathrm{n}=265)$ as compared with the global integrated analysis $(n=2,041) .{ }^{16}$ Additionally, when comparing the AE pattern with the global pooled population, no differentiation between genotypes was considered, and data from all genotypes (GT1-6) were included. Furthermore, the present analysis in the Korean population included a small number of high-risk patients such as previous injection drug users (IDU; 5.7\%), those with sofosbuvir-based treatment-experience (1.9\%), compensated cirrhosis (14.0\%), HIV coinfection (13.2\%), and non-CC interleukin 28B subtype (23.0\%). Most of the population were not previous IDUs (94.3\%, 250/265), which may have resulted in better treatment adherence and compliance than in the global 
integrated analysis $(61 \%, 1,243 / 2,041) .{ }^{16}$ Nevertheless, a recent pooled analysis of seven phase III trials evaluating efficacy and safety of 8-week or 12-week G/P treatment demonstrated that treatment adherence and compliance in recent IDUs were high despite enduring concerns. ${ }^{28}$ The current international guidelines (European Association for the Study of the Liver ${ }^{2}$ and American Association for the Study of Liver Diseases ${ }^{12}$ ) emphasize that treatment response among IDUs is similar to that in non-IDU populations. Moreover, this high-risk group could benefit from a shorter treatment duration (8 weeks). ${ }^{28}$ Interleukin 28B CC genotype, which is estimated to be present in around $90 \%$ of the Korean population, has been strongly associated with spontaneous viral clearance and favorable clinical outcomes. ${ }^{29}$ However, it is still unclear whether Interleukin 28B genotype has a strong influence on the effects of DAAs, or which DAAs are best for people with non-CC genotypes.

In conclusion, results from this analysis indicate that treatment with G/P combination therapy for 8 or 12 weeks is highly efficacious and well tolerated in Korean patients with HCV GT1 or GT2 infections, supporting the current Korean Association for the Study of the Liver recommendation. ${ }^{11}$

\section{CONFLICTS OF INTEREST}

Y.S.L. is an advisory board member of Bayer Healthcare and Gilead Sciences. E.D.C., L.M.F., and N.N.A. are employees of AbbVie, Inc. and may hold stock or stock options. A.A. and D.H.K. are former employees of AbbVie, Inc. and may hold stock or stock options. Other authors have no conflicts of interest to declare.

Y.J.K. is an editorial board member of the journal but was not involved in the peer reviewer selection, evaluation, or decision process of this article. No other potential conflicts of interest relevant to this article were reported.

\section{ACKNOWLEDGMENTS}

The design, study conduct, analysis, and financial support of the trials (ENDURANCE 1, ENDURANCE 2, SURVEYOR II part 4, VOYAGE I and VOYAGE II) was provided by AbbVie. AbbVie participated in the interpretation of data, review, and approval of the publication. All authors had access to all relevant data.

The authors would like to express their gratitude to the trial participants, investigators, and coordinators who made this study possible. Medical writing assistance was provided by Syed Abdul Haseeb and Julia Ventura from MediTech Media (Singapore), which was funded by AbbVie in accordance with Good Publication Practice guidelines.

\section{AUTHOR CONTRIBUTIONS}

Study design: D.H.K., E.D.C. Formal analysis: E.D.C., L.M.F., N.N.A., A.A. Study conduction and data collection: J.H., Y.J.K., J.W.L., J.H.K., Y.S.L., K.H.H., S.H.J., M.C., K.T.Y., S.H.B., S.W.P., Y.J.L. Data Interpretation, review, approval of final manuscript: all authors.

\section{ORCID}

Jeong Heo https://orcid.org/0000-0003-0961-7851 Yoon Jun Kim Jin-Woo Lee https://orcid.org/0000-0001-9141-7773 https://orcid.org/0000-0002-7227-4938 Ji Hoon Kim https://orcid.org/0000-0002-4427-3997 Young-Suk Lim https://orcid.org/0000-0002-1544-577X Kwang-Hyub Han https://orcid.org/0000-0003-3960-6539 Sook-Hyang Jeong https://orcid.org/0000-0002-4916-7990 Mong Cho https://orcid.org/0000-0002-0498-6300 Ki Tae Yoon https://orcid.org/0000-0002-8580-0239 Si Hyun Bae https://orcid.org/0000-0003-1727-7842 Eric D. Crown https://orcid.org/0000-0002-5157-1095 Linda M. Fredrick https://orcid.org/0000-0001-7596-0784 Negar Niki Alami https://orcid.org/0000-0003-4455-5780 Armen Asatryan https://orcid.org/0000-0003-2812-4243 Do Hyun Kim https://orcid.org/0000-0003-0064-1732 Seung Woon Paik https://orcid.org/0000-0002-6746-6652 Youn-Jae Lee https://orcid.org/0000-0003-3854-3388

\section{REFERENCES}

1. World Health Organization (WHO). Global hepatitis report, 2017 [Internet]. Geneva: WHO; c2017 [cited 2020 Oct 28]. Available from: https://www.who.int/hepatitis/publications/ global-hepatitis-report2017/en/.

2. European Association for the Study of the Liver. European Association for the Study of the Liver. EASL Recommendations on Treatment of Hepatitis C 2018. J Hepatol 2018;69: 461-511.

3. Forns X, Lee SS, Valdes J, et al. Glecaprevir plus pibrentasvir for chronic hepatitis $C$ virus genotype $1,2,4,5$, or 6 infection in adults with compensated cirrhosis (EXPEDITION-1): a single-arm, open-label, multicentre phase 3 trial. Lancet Infect Dis 2017;17:1062-1068. 
4. Messina JP, Humphreys I, Flaxman A, et al. Global distribution and prevalence of hepatitis $\mathrm{C}$ virus genotypes. Hepatology 2015;61:77-87.

5. Xu F, Moorman AC, Tong X, et al. All-cause mortality and progression risks to hepatic decompensation and hepatocellular carcinoma in patients infected with hepatitis $\mathrm{C}$ virus. Clin Infect Dis 2016;62:289-297.

6. Cho EJ, Jeong SH, Han BH, Lee SU, Yun BC, Park ET. Hepatitis $\mathrm{C}$ virus (HCV) genotypes and the influence of $\mathrm{HCV}$ subtype $1 \mathrm{~b}$ on the progression of chronic hepatitis $\mathrm{C}$ in Korea: a single center experience. Clin Mol Hepatol 2012;18:219224.

7. Jeong SH. Epidemiology of hepatitis C virus infection in Korea. Korean J Med 2015;88:630-634.

8. Jeong SH, Jang ES, Choi HY, Kim KA, Chung W, Ki M. Current status of hepatitis $\mathrm{C}$ virus infection and countermeasures in South Korea. Epidemiol Health 2017;39:e2017017.

9. Korean Association for the Study of the Liver. KASL clinical practice guidelines: management of hepatitis C. Clin Mol Hepatol 2016;22:76-139.

10. Uyanikoglu A, Kaymakoglu S, Danalioglu A, et al. Durability of sustained virologic response in chronic hepatitis C. Gut Liver 2013;7:458-461.

11. Yeon JE. Recent update of the 2017 Korean Association for the Study of the Liver (KASL) treatment guidelines of chronic hepatitis $\mathrm{C}$ : comparison of guidelines from other continents, 2017 AASLD/IDSA and 2016 EASL. Clin Mol Hepatol 2018;24:278-293.

12. AASLD-IDSA HCV Guidance Panel. Hepatitis C guidance 2018 update: AASLD-IDSA recommendations for testing, managing, and treating hepatitis $\mathrm{C}$ virus infection. Clin Infect Dis 2018;67:1477-1492.

13. Asselah T, Boyer N, Saadoun D, Martinot-Peignoux M, Marcellin P. Direct-acting antivirals for the treatment of hepatitis $\mathrm{C}$ virus infection: optimizing current IFN-free treatment and future perspectives. Liver Int 2016;36 Suppl 1:47-57.

14. European Medicines Agency. Maviret summary of product characteristics: glecaprevir/pibrentasvir $100 \mathrm{mg} / 40 \mathrm{mg}$ filmcoated tablets. Amsterdam: European Medicines Agency; c2020 [cited 2020 Oct 28]. Available from: https://www.ema. europa.eu/.

15. AbbVie Inc. Application for inclusion of Maviret (glecaprevir/pibrentasvir) on the WHO Model List of Essential Medicines (EML). Geneva: World Health Organization; c2019 [cited 2020 Oct 28]. Available from: https://www.who. int/selection_medicines/committees/expert/22/applications/ s6.4.4.2_glecprevir-pibrentasvir.pdf?ua=1.

16. Puoti M, Foster GR, Wang S, et al. High SVR12 with 8-week and 12-week glecaprevir/pibrentasvir therapy: an integrated analysis of HCV genotype 1-6 patients without cirrhosis. J Hepatol 2018;69:293-300.
17. Brown RS Jr, Buti M, Rodrigues L, et al. Glecaprevir/pibrentasvir for 8 weeks in treatment: naive patients with chronic HCV genotypes 1-6 and compensated cirrhosis. The EXPEDITION-8 trial. J Hepatol 2020;72:441-449.

18. Nam JY, Jang ES, Kim YS, et al. Epidemiological and clinical characteristics of hepatitis $\mathrm{C}$ virus infection in South Korea from 2007 to 2017: a prospective multicenter cohort study. Gut Liver 2020;14:207-217.

19. Naganuma A, Chayama K, Notsumata K, et al. Integrated analysis of 8-week glecaprevir/pibrentasvir in Japanese and overseas patients without cirrhosis and with hepatitis $C$ virus genotype 1 or 2 infection. J Gastroenterol 2019;54:752-761.

20. Zeuzem S, Foster GR, Wang S, et al. Glecaprevir-pibrentasvir for 8 or 12 weeks in HCV genotype 1 or 3 infection. N Engl J Med 2018;378:354-369.

21. Asselah T, Kowdley KV, Zadeikis N, et al. Efficacy of glecaprevir/pibrentasvir for 8 or 12 weeks in patients with hepatitis C virus genotype 2, 4, 5, or 6 infection without cirrhosis. Clin Gastroenterol Hepatol 2018;16:417-426.

22. Wei L, Wang G, Alami NN, et al. Glecaprevir-pibrentasvir to treat chronic hepatitis $\mathrm{C}$ virus infection in Asia: two multicentre, phase 3 studies. A randomised, double-blind study (VOYAGE-1) and an open-label, single-arm study (VOYAGE-2). Lancet Gastroenterol Hepatol 2020;5:839-849.

23. Park SH, Park CK, Lee JW, et al. Efficacy and tolerability of peginterferon alpha plus ribavirin in the routine daily treatment of chronic hepatitis $\mathrm{C}$ patients in Korea: a multi-center, retrospective observational study. Gut Liver 2012;6:98-106.

24. Lee HW, Oh SR, Kim DY, et al. Daclatasvir plus asunaprevir for the treatment of patients with hepatitis $\mathrm{C}$ virus genotype 1b infection: real-world efficacy, changes in liver stiffness and fibrosis markers, and safety. Gut Liver 2018;12:324-330.

25. Younossi ZM, Stepanova M, Henry L, et al. Sofosbuvir and ledipasvir are associated with high sustained virologic response and improvement of health-related quality of life in East Asian patients with hepatitis C virus infection. J Viral Hepat 2018;25:1429-1437.

26. Cho Y, Cho EJ, Lee JH, Yu SJ, Yoon JH, Kim YJ. Sofosbuvirbased therapy for patients with chronic hepatitis C: early experience of its efficacy and safety in Korea. Clin Mol Hepatol 2015;21:358-364.

27. Kapadia SN, Marks KM. Hepatitis C management simplification from test to cure: a framework for primary care providers. Clin Ther 2018;40:1234-1245.

28. Foster GR, Dore GJ, Wang S, et al. Glecaprevir/pibrentasvir in patients with chronic $\mathrm{HCV}$ and recent drug use: an integrated analysis of 7 phase III studies. Drug Alcohol Depend 2019;194:487-494.

29. Kil H, Jeong SH, Kim JW, et al. Role of interleukin-28B genetic polymorphisms in Korean patients with hepatitis $\mathrm{C}$ virus infection. Gut Liver 2014;8:70-78. 